\title{
OsAM1 is required for leptotene-zygotene transition in rice
}

\author{
Lixiao Che ${ }^{1, *}$, Ding Tang ${ }^{1, *}$, Kejian Wang ${ }^{1}$, Mo Wang ${ }^{1}$, Keming $\mathrm{Zhu}^{2}$, Hengxiu $\mathrm{Yu}^{2}$, Minghong $\mathrm{Gu}^{2}$, \\ Zhukuan Cheng ${ }^{1}$
}

${ }^{I}$ State Key Laboratory of Plant Genomics and Center for Plant Gene Research, Institute of Genetics and Developmental Biology, Chinese Academy of Sciences, Beijing 100101, China; ${ }^{2}$ Key Laboratory of Plant Functional Genomics of Ministry of Education, Yangzhou University, Yangzhou 225009, China

The events occurring at the onset of meiosis have not been fully elucidated. In the present study, $O$ s $A M 1$ was identified in rice (Oryza sativa L.) by map-based cloning. OsAM1, a homolog of Arabidopsis SWI1 and maize AM1, encodes a protein with a coiled-coil domain in its central region. In the Osam 1 mutant, pollen mother cells are arrested at leptotene, showing that OsAM1 is required for the leptotene-zygotene transition. Immunocytological analysis revealed that OsAM1 exists as foci in early prophase I meiocytes. Very faint OsREC8 foci persisted in the Osam1 mutant, indicating that OsAM1 is not required for the initial meiotic recruitment of OsREC8. In the absence of OsAM1, many other critical meiotic components, including PAIR2, ZEP1 and OsMER3, could not be correctly installed onto chromosomes. In contrast, in pair2, Osmer 3 and zep1 mutants, OsAM1 could be loaded normally, suggesting that OsAM1 plays a fundamental role in building the proper chromosome structure at the beginning of meiosis.

Keywords: rice; meiosis; leptotene-zygotene transition; OsAM1

Cell Research (2011) 21:654-665. doi:10.1038/cr.2011.7; published online 11 January 2011

\section{Introduction}

Eukaryotic meiosis is a highly coordinated cell division process, generating the haploid cells required for sexual reproduction. In higher plants, these haploid reproductive cells then develop into gametophytes after mitotic divisions and yield pollen and ovules. After fertilization, the chromosome number of embryos returns to that of their parents [1]. Therefore, meiosis is important for sexually reproducing organisms. It facilitates the maintenance of the stable chromosome number of a species, and more importantly produces genetic variations in gametes, which in turn promote genetic and phenotypic variations in its offspring [2].

Meiosis involves one round of DNA replication followed by two rounds of cell division: meiosis I and meiosis II. The meiotic cell cycle is thought to start at pre-meiotic S phase [3], a highly dynamic stage of early

\footnotetext{
*These two authors contributed equally to this work.

Correspondence: Zhukuan Cheng

Tel: +86-10-64868726; Fax: +86-10-64873428

E-mail: zkcheng@genetics.ac.cn

Received 5 May 2010; revised 19 August 2010; accepted 16 September 2010; published online 11 January 2011
}

prophase I during which several critical events related to meiotic chromosome structure take place, including formation of sister chromatid cohesion (SCC) and axial elements (AE) [4]. It is only after these early events have occurred that the subsequent steps, namely, homology search, recombination, pairing and assembly of the synaptonemal complex (SC) can proceed successfully.

Meiosis I is critical for its reductional pattern of chromosome segregation. Cohesin proteins, which are thought to be involved in this process, have been extensively studied in yeast and animals [4-6]. In plants, some cohesin proteins have been characterized for their influence in meiotic pairing, especially the REC8 homologs: SYN1/DIF1 in Arabidopsis [7-9], AFD1 in maize [10] and OsRAD21-4 in rice [11]. It is becoming evident that the cohesin complex provides the basis for AE assembly and is implicated in various meiotic events $[4,12]$.

In early meiosis, immediately following the SCC formation, the chromatids undertake the process of homology search and pairing, a process that is both fundamental and unique to a faithful meiotic division [4]. There are many lines of evidence indicating that early recombination intermediates are involved in the homology search $[13,14]$. In maize phs 1 (poor homologous synapsis 1) meiocytes, loading of RAD51 complexes that catalyze 
the single-strand invasion step in recombination is greatly inhibited and results in synapsis among non-homologues [15]. Moreover, in many species, an interesting pre-synaptic configuration termed the chromosome bouquet, in which all telomeres are spatially attached to the nucleus envelope and clustered within a limited area, is thought to facilitate the homology search [16]. The paml (plural abnormalities of meiosis 1) mutant of maize, which is defective in telomere clustering, has normal RAD51 foci on zygotene chromosomes, but shows aberrant synapsis and a dramatic reduction in homologous pairing [17]. However, the clustering of telomeres may only be one of the possible mechanisms that facilitate initial homology recognition and, thus, may be evolutionarily dispensable. In Sordaria, homologs have completed homology search before the bouquet formation, and the maize phs 1 mutation has little effect on bouquet formation in $\sim 50 \%$ of the meiocytes. Moreover, Caenorhabditis elegans and Drosophila lack a bouquet altogether, but can undergo complete meiosis [16].

Although an abundance of components controlling the meiotic processes have been identified, little is known about what underlies the initiation of meiosis. In organisms with facultative meiosis, such as yeast, the decision of whether to initiate meiosis is made before the onset of the pre-meiotic $S$ phase $[3,18]$. In multicellular organisms, meiosis occurs in cells that are already destined to become meiocytes, and the switch from mitosis to meiosis is also under genetic control. In mammals, mouse STRA8 regulates retinoic acid signaling, which has the ability to induce meiosis, and germ cells of the STRA8knockout mouse arrest before pre-meiotic $S$ phase [19]. The isolation of two genes, SWI1 (SWITCH1) in Arabidopsis and its homolog, AM1 (AMEIOTIC1) in maize, has shed more light on this question in plants.

In swi 1-2 mutant male meiocytes, chromatid arms and centromeres lose their cohesion, resulting in the formation of 20 chromatids instead of 5 bivalents; however, female meiocytes retain centric/whole-arm cohesion, but lack synapsis and undergo a mitosis-like division in lieu of a reductional meiosis I [20, 21]. In the am1-1 (ameiotic1-1) mutant allele of maize, both male and female meiocytes fail to enter meiosis; instead, they proceed with several mitotic divisions and then degrade. Plants containing the am1-praI (ameiotic1-prophase I arrest) allele enter meiotic prophase and are arrested at the leptotene-zygotene transition [22-24]. Both genes appear to be required for early meiotic events, e.g., for SCC and initiation of synapsis. AM1 accumulation starts during the pre-meiotic interphase and SWI1 is detected early at pre-meiotic G1 phase [25], which place them as the earliest acting meiotic proteins in plants identified yet to date.
Compared with yeast, Arabidopsis and maize, the study of rice meiotic networks has lagged behind [26], and only a few genes have been identified [27-33]. Among them is a recently cloned gene MEL1 (MEIOSIS ARRESTED AT LEPTOTENE 1), which controls the progression of meiosis. In mell meiocytes, meiosis is arrested early in prophase I and chromosomes frequently show an uncondensed morphology, similar to leptotene or zygotene. Chromatin modification is altered and abnormally dense PAIR2 signals accumulate in the nucleolus, implying that MEL1 may insulate rDNA repeats from homologous synapsis and recombination to maintain the structure of heterochromatin or nucleolar-organizing regions. MEL1 may also function in maintaining the germ cell identity by repressing the ectopic expression of somatic genes [31].

Here, we report two allelic rice mutants, Osam 1-1 and Osam 1-2, in which the meiotic process of male meiocytes also appears to be arrested at early prophase $\mathrm{I}$, resulting in a completely sterile phenotype. Utilizing a combination of genetic and immunocytological analyses, we carried out a detailed study of the two mutant alleles, and showed that a series of interdependent key meiotic events were disrupted in the mutants. Our results are expected to add new insights into the complex network of meiosis events in rice.

\section{Results}

\section{Identification of the Osam1-1 mutant}

A completely sterile mutant derived from a japonica cultivar Wuxiangjing 9 was identified from the M1 populations induced by ethyl methane sulfonate (EMS). The

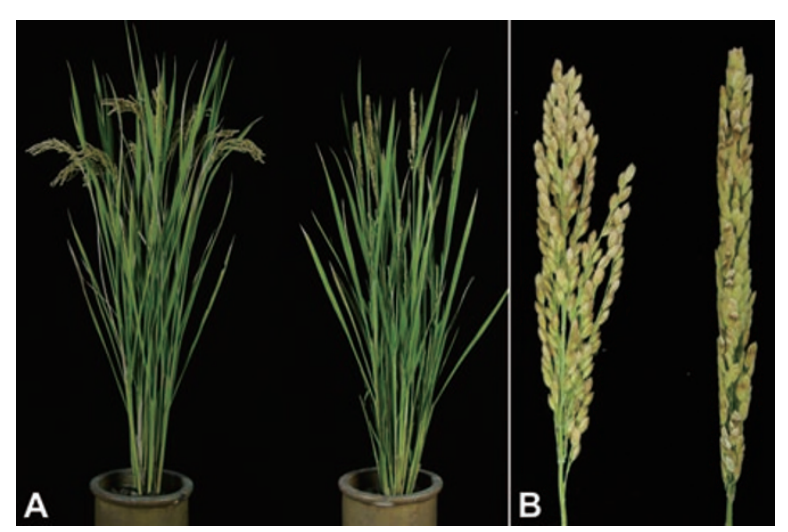

Figure 1 Comparison of the wild-type and Osam1-1 mutant phenotype. (A) A wild-type plant (left) and an Osam1-1 mutant plant (right). (B) A wild-type panicle (left) and an Osam1-1 mutant panicle (right). 


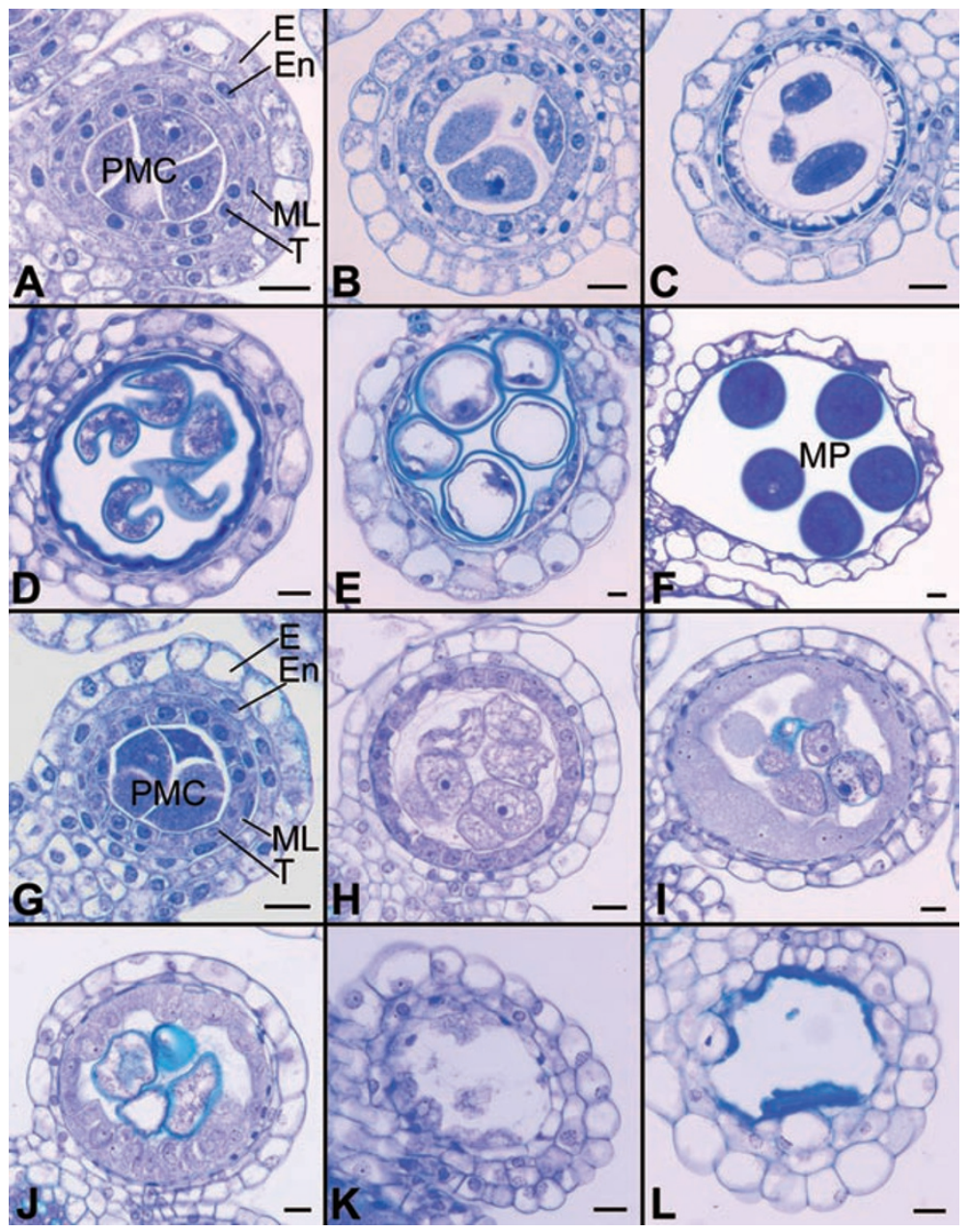

Figure 2 Transverse sections of anthers in both wild type and the Osam1-1 mutant. As the development of the Osam1-1 PMCs was arrested, the sections of Osam1-1 anthers were selected according to the length of spikelet. The wild-type sections are shown in $\mathbf{A}-\mathbf{F}$, and the Osam1-1 sections are shown in $\mathbf{G}-\mathbf{L}$. (A, B, G, H) Early meiosis stage. (C, I) Late meiosis stage. (D, J) Vacuolated pollen stage. (E, K) Pollen mitosis stage. (F, L) Mature pollen stage. E, epidermis; En, endothecium; ML, middle layer; T, tapetum; PMCs, pollen mother cells; MP, mature pollen. Scale bars, $5 \mu \mathrm{m}$.

mutant plant was normal in vegetative development, although no pollen was produced (Figure 1A and 1B). Normal phenotype plants and sterile plants from the progeny of Osam 1+/- give 3:1 segregation, establishing it as a single recessive mutant $\left(\chi^{2}=0.83 ; P>0.05\right)$. We named the mutant Osam1-1. When pollinated with normal pollen from wild-type plants, Osam 1-1 plants do not set seeds. Thus, macrospore development is also affected, but it was not studied further.

To determine the anther morphological defects in $O_{S}$ am 1-1, transverse sections of both wild-type and mutant anthers at different developmental stages were examined. The development of rice anthers has been described in detail $[34,35]$. Before entering meiosis, the four-layered anther wall, including epidermis, endothecium, middle layer and tapetum, is formed and primary sporogenous cells develop into larger PMCs in the anther locules (Figure 2A). The PMCs gradually become spherical and enter meiosis (Figure 2B and 2C). At the end of meiosis, the PMCs develop into tetrads. Concomitantly, the middle layer and tapetum begin to degenerate. After going through the vacuolated stage (Figure 2D) and two rounds of mitosis (Figure 2E), microspores then generate the mature tricellular pollen grains (Figure 2F).

There was no detectable difference between Osam 1-1 and wild-type anthers before entering meiosis, and the four layers of anther wall were also normal (Figure 2G). The impairment of anther development was observed in Osam 1-1 after PMCs enlarged and entered meiosis (Figure $2 \mathrm{H}$ ). The tapetal cells had significantly swelled, 
and the PMCs did not complete meiosis (Figure 2I). The development of PMCs in Osam 1-1 anthers seemed to be interrupted; they were arrested at the PMC stage with aberrant tapetum development (Figure 2J and 2K). Eventually, all the PMCs as well as tapetum had disappeared, leading to the anthers without pollen (Figure 2L).

\section{Cloning of the OsAM1 gene}

The OsAM1 gene was isolated using a map-based cloning strategy. We constructed the mapping population by crossing heterozygous $O s a m 1^{+/-}$to an indica cultivar Nanjing 11. OsAM1 was first mapped to the long arm of chromosome 3, and then further narrowed to a $120 \mathrm{~kb}$ region. From the Rice Genome Annotation Project database (http://rice.plantbiology.msu.edu/cgi-bin/gbrowse/ rice), we selected one candidate gene (Os03g44760) annotated as SWI1, which shows high similarity with AMEIOTIC1 from maize and SWITCH1 from Arabidopsis. When we sequenced this candidate gene, we found a $\mathrm{C}$ to $\mathrm{T}$ point mutation in Osam 1-1, relative to wild type, which caused an arginine-to-tryptophan amino-acid substitution at peptide position 360 .

We also identified a second mutant with the same phenotype and named it Osam 1-2. It was obtained from an indica rice variety Zhongxian 3037, induced by ${ }^{60} \mathrm{Co} \sim \gamma$ ray radiation. Sequence analysis showed a two-nucleotide deletion in the second exon of the OSAMI gene introduc- ing a premature stop codon. Additionally, we generated a gene-specific p35S OSAM1-RNAi construct to confirm that the mutant phenotype was attributed to the loss of function of OsAM1. The OsAM1-RNAi lines showed severe reduction of fertility, and their chromosome behavior in PMCs was identical to that of Osam1-1 (Supplementary information, Figure S1A and S1B). In the RNAi plants, the transcript level of OSAM1 was found to be greatly reduced, as determined by RT-PCR analysis (Supplementary information, Figure S1C). From these data, we conclude that this locus encodes the OSAMI gene.

\section{Structure of the OsAM1 gene and the corresponding pro- tein}

There are two full-length cDNAs of the OSAM1 gene, AK101644 and AK064367, from the public network database. By comparing the two sequences, we found that the seventh exon of AK101644 (63 bp) was absent in AK064367. Both of the sequences were shown to be true transcripts by RT-PCR, and they may have undergone alternative splicing. However, the abundance of the larger transcript was much higher.

To examine the expression pattern of OsAM1, RT-PCR assays were conducted on the total RNA extracted from root, stem, leaf, panicle and seedling tissues. We found that OsAM1 is expressed in all these tissues (Supplementary information, Figure S2).

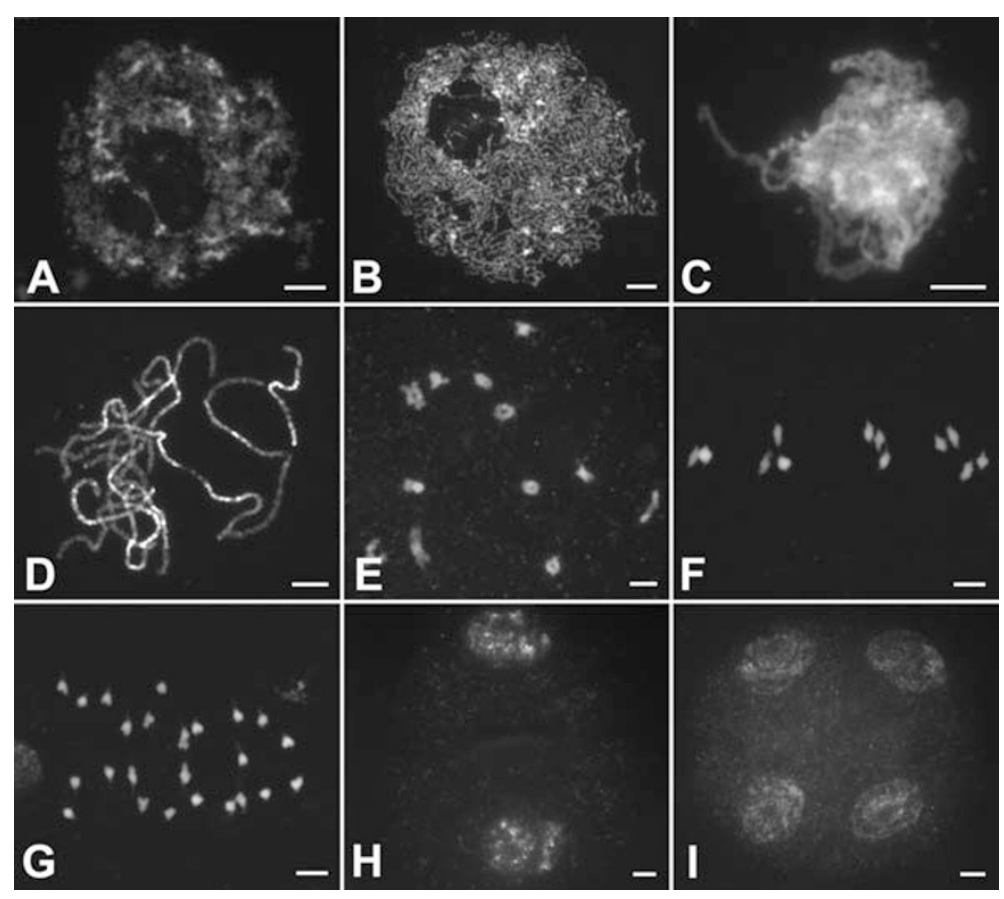

Figure 3 Meiosis of male meiocytes in wild type. (A) Early leptotene; (B) late leptotene; (C) zygotene; (D) pachytene; (E) diakinesis; (F) metaphase I; (G) anaphase I; (H) dyad; and (I) Tetrad. Scale bars, $5 \mu \mathrm{m}$. 
Alignment of the larger full-length cDNA sequence with the genomic sequence shows that the OsAM1 gene is composed of 10 exons and 9 introns (Supplementary information, Figure S3). OsAM1 encodes an 803-aminoacid product, with a molecular weight of $88.2 \mathrm{kDa}$. From a database search using BLASTp, the OsAM1 protein shows $61 \%$ identity to maize AMEIOTIC1, and $34 \%$ identity to Arabidopsis SWITCH1. A multiple-alignment analysis revealed that the most conserved region lies in the middle of the polypeptide; the amino-acid substitution that occurred in Osam 1-1 is also located in this region (Supplementary information, Figure S4). Additionally, OsAM1 contains a predicted coiled-coil domain (449-567 amino acids) (SMART, http://smart.emblheidelberg.de), suggesting its potential of associating with other meiotic factors.

\section{Meiotic course is arrested at leptotene in the Osam $1 \mathrm{mu}$ - tants}

In wild-type PMCs, early leptotene chromosomes begin to condense and individual chromosomes appear as thin threads (Figure 3A), while nucleoli move to the periphery of the nuclei in late leptotene (Figure 3B). As zygotene progresses, homologous chromosomes undergo pairing and synapsis (Figure 3C). At pachytene, fully synapsed chromosomes are observed (Figure 3D). SCs are then disassembled, and chiasmata, which correspond to crossovers, hold the homologous chromosomes together at diplotene. During diakinesis, the chromosomes are highly condensed and 12 short bivalents are clearly
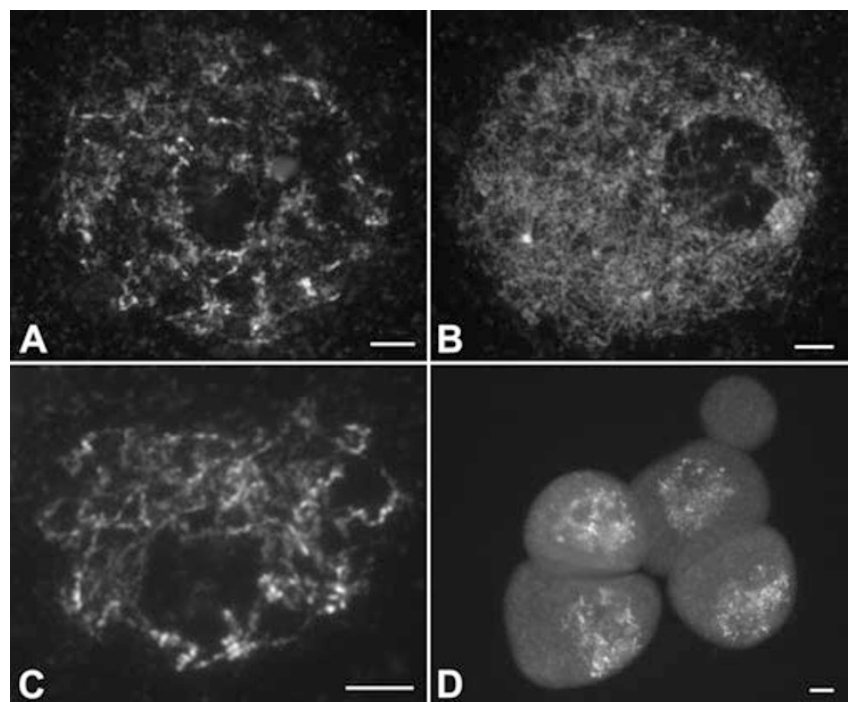

Figure 4 Meiosis of male meiocytes in the Osam1-1 mutant. (A) Early leptotene. (B and C) Late leptotene. (D) The arrested PMCs. Scale bars, $5 \mu \mathrm{m}$.

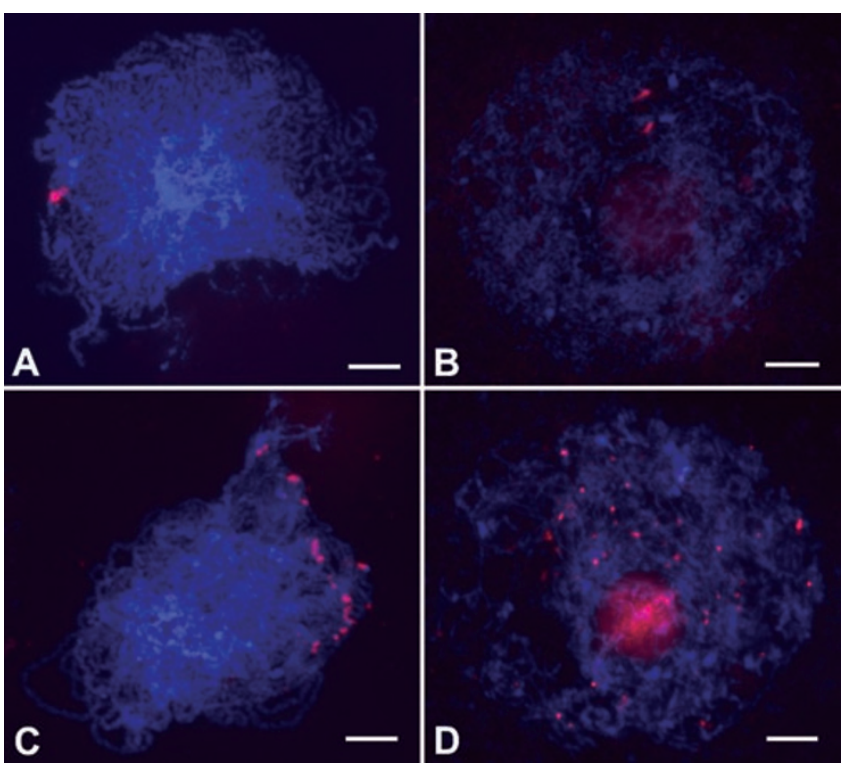

Figure 5 Detection of homologous chromosome pairing and telomere bouquet formation. (A, B) Chromosomes probed with 5S rDNA in wild type (A) and the Osam1-1 mutant (B). (C, D) Telomere bouquet formation revealed by FISH using pAtT4 as the probe in wild type (C) and the Osam1-1 mutant (D). Chromosomes are stained with DAPI. Scale bars, $5 \mu \mathrm{m}$.

observed (Figure 3E). At metaphase I, the bivalents become aligned in the center of the cell (Figure 3F), and then homologous chromosomes separate and migrate toward opposite poles at anaphase I (Figure 3G), generating dyads at the end of meiosis I. During meiosis II, the two dyads undergo equational division and produce tetrads (Figure $3 \mathrm{H}$ and $3 \mathrm{I}$ ).

The Osam 1-1 chromosomes behaved normally during meiotic interphase and leptotene (Figure 4A and 4B). However, the later stages of prophase I were never observed; the meiotic process seemed to have been arrested at leptotene, although some chromosomes continue to condense (Figure 4C). The abnormal PMCs remained in leptotene for some time (Figure 4D). We have checked more than 300 PMCs in the Osam 1-1 mutant and all of them showed the same characteristics. We also found that Osam1-2 meiosis is identical to Osam1-1.

\section{Homologous chromosome pairing is absent in Osam1-1}

To discriminate the behavior of chromosomes between wild type and the Osam1-1 mutant, we performed fluorescent in situ hybridization (FISH) using 5S rDNA as the probe in both wild-type and Osam1-1 PMCs. 5S rDNA has a single locus in the rice genome, located on the short arm of chromosome 11 [36]. In wild-type meiocytes, two unpaired 5S rDNA signals were visible at lep- 
totene. At zygotene, only one bright signal was observed in each cell, showing that the homologous chromosomes paired well at this stage (Figure 5A). However, two separate signals were always visible in Osam 1-1 meiocytes (Figure 5B), indicating that the homologous chromosome pairing is perturbed in this mutant.

Telomere bouquet formation is absent in Osam 1-1 PMCs

An important feature of meiosis is that homologous chromosomes search for each other, pair and synapse along their entire length. Telomere bouquet clustering, an event specific to early meiotic prophase, increases the efficiency of these processes. To investigate telomere bouquet formation in wild type and Osam 1-1, we probed meiotic chromosomes at leptotene with the clone pAtT4 that contains telomeric repeats. In wild type, almost all telomeres were adjacent to the nuclear envelope and clustered, showing a typical bouquet configuration (Figure 5C). However, in Osam1-1, the telomeres scattered throughout the nucleus randomly; no bouquet was formed (Figure 5D), further showing that the PMC development had halted at leptotene.

\section{Meiotic recombination initiation occurs in Osam 1}

Meiotic recombination is initiated by double-strand breaks (DSBs) that occur between leptotene and zygotene [4]. To investigate whether recombination occurs in Osam 1-1, antibodies that recognize the phosphorylated
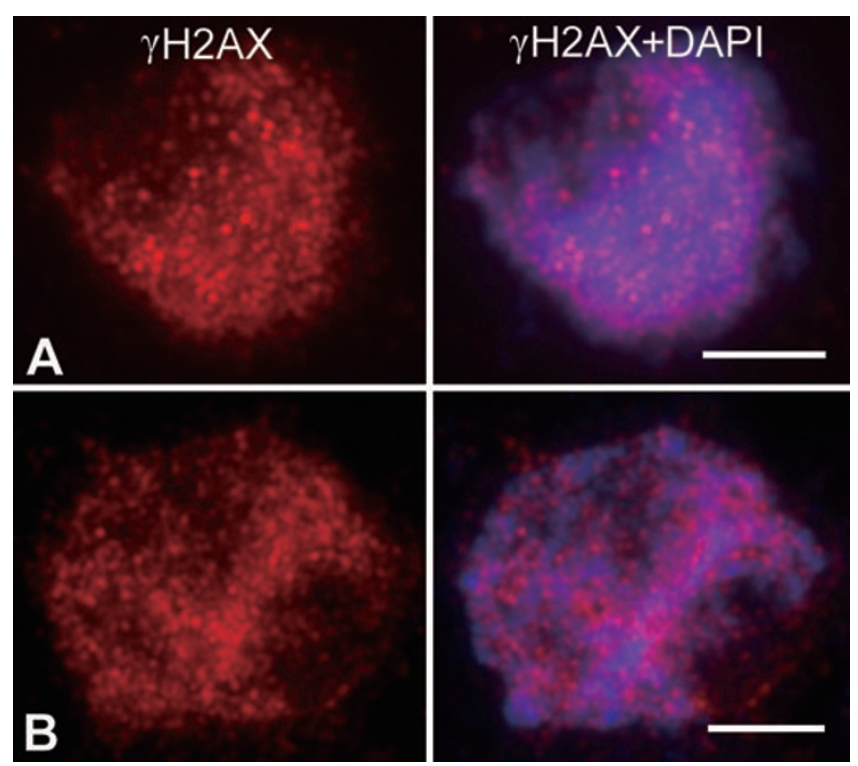

Figure 6 Detection of DNA DSBs formation in wild-type (A) and Osam1-1 (B) PMCs. Chromosomes were shown by DAPI (blue) staining and DSBs were shown by anti- $\gamma \mathrm{H} 2 \mathrm{AX}$ staining (red). Scale bars, $5 \mu \mathrm{m}$. form of the histone variant $\mathrm{H} 2 \mathrm{AX}(\gamma \mathrm{H} 2 \mathrm{AX})$ were used to monitor DNA DSBs formation $[37,38]$. In wild-type PMCs, $\gamma \mathrm{H} 2 \mathrm{AX}$ foci were apparent beginning at leptotene. During zygotene, the $\gamma \mathrm{H} 2 \mathrm{AX}$ foci number reached its maximum (Figure 6A), while in Osam 1-1 PMCs, strong $\gamma \mathrm{H} 2 \mathrm{AX}$ signals were also visible, implying that the normal level of DSBs had occurred (Figure 6B). The same result was obtained in Osam 1-2. Thus, we suggest that the meiotic recombination is initiated normally in Osam1.

\section{OsAM1 functions at early prophase I}

To further examine the role of OsAM1 during meiosis, antibodies were raised against the $\mathrm{N}$-terminal region (amino acids 21-91) of the OsAM1 protein. Western blot analysis showed that the OsAM1 protein was present in young panicles, but not in vegetative organs (Supplementary information, Figure S5), suggesting that OSAM1 expression is subjected to regulation at the translational level.

For further studies, a dual immunolocalization experiment was performed in PMCs of wild type, using antibodies against OsREC8 and OsAM1. In budding yeast, REC8 is believed to be a factor belonging to the meiotic cohesin complex [5]. Its homologs also exist in maize, Arabidopsis and rice. The nuclear pattern of OsREC8 was identified recently in rice [29]. In the present study, OsREC8 labeling was first visible as punctated foci in pre-meiotic interphase nuclei (Figure 7A). These foci were then loaded onto the chromosomes at leptotene (Figure 7B). At zygotene, thicker OsREC8 signals delineated the chromosome axes, and the entire chromosome sets appeared as a crescent (Figure 7C). During pachytene, the OsREC8 signals were distributed among all paired chromosomes (Figure 7D and 7E). At diplotene and diakinesis, OsREC8 signals on chromosomes began to decrease, while the chromosomes shrank further. Therefore, OsREC8 can be used as a chromosome marker to monitor meiotic events during early prophase I.

OsAM1 foci, like OsREC8, were also detected at pre-meiotic interphase and they appeared to be diffused signals (Figure 7A). At leptotene, the number of OsAM1 foci increased rapidly (Figure 7B). During the two stages, OsAM1 and OsREC8 foci did not overlap well. At zygotene, the intensity of OsAM1 signals continued to increase and reached its peak (Figure 7C); however, they still remained dot-like and never became linear. At early pachytene, the OsAM1 staining on chromosomes began to diminish, whereas those in the nucleoplasm increased (Figure 7D). Subsequently, the OsAM1 signals quickly diminished as the SCs elongated, and they almost disappeared in the nucleus after pachytene (Figure 7E). This 


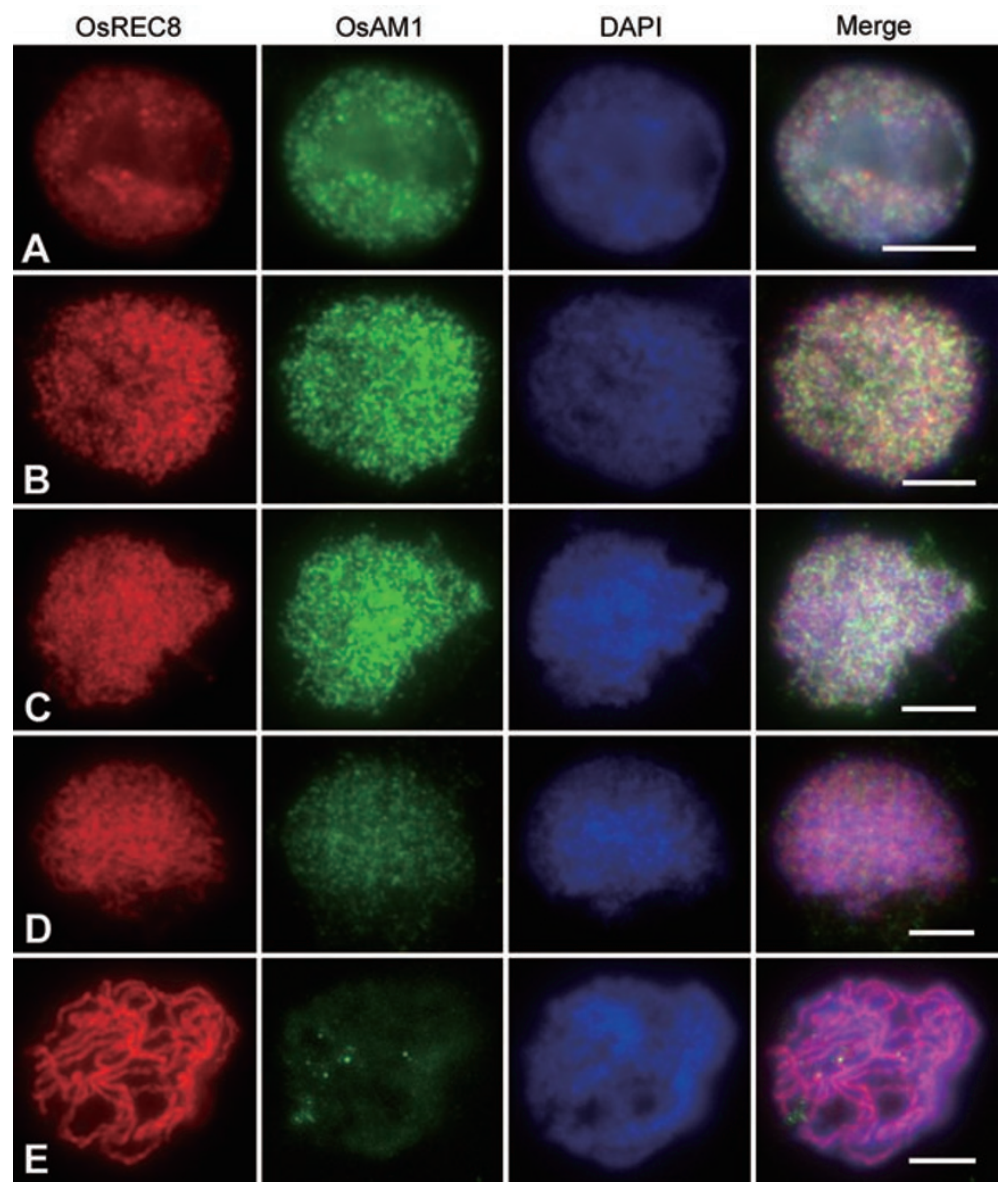

Figure 7 Dual immunolocalization of OsREC8 and OsAM1 in wild-type rice. Panels from left to right indicate the localization of OsREC8, the localization of OsAM1, DAPI staining and the merged image, respectively. (A) Pre-meiotic interphase; (B) leptotene; (C) zygotene; (D) early pachytene; and (E) pachytene. Scale bars, $5 \mu \mathrm{m}$.

pattern suggests that OsAM1 is an early meiosis factor, and its removal occurs much earlier than that of OsREC8.

\section{Meiotic element installation is disrupted in Osam1-1}

Improper installation of meiotic elements can cause serious impairments during meiosis. In the present study, we found all PMCs of the two Osam 1 alleles were arrested at leptotene. Thus, it is important to study whether loss of OsAM1 has any influence on the installation of other meiotic elements. The anti-OsAM1 antibody was used to determine whether the mutation affected the localization of OsAM1 protein. Antibodies against several other proteins involved in meiosis, including OsREC8, PAIR2, OsMER3 and ZEP1, were used to question whether their patterns were altered in Osam1-1. Among them, PAIR2 is the rice homolog of $S$. cerevisiae HOP1 and Arabidopsis ASY1, which associates with AEs at leptotene and zygotene and is removed from the AEs when homologous chromosomes have synapsed [39]. The distribution pattern of PAIR2 reflects the status of the chromosome axes. OsMER3 is a rice ZMM protein that is required for the formation of crossovers; it can mark the installation of recombination elements [29]. ZEP1 is thought to be a component of CEs in SCs like ZIP1 in S. cerevisiae and ZYP1 in Arabidopsis [33]. To be sure we were looking at PMCs rather than somatic cells, we used a fluorescently labeled anti-CENH3 antibody to label the centromeres. The anti-CENH3 signal from the centromeres in meiocytes is characteristically brighter than the signal observed from somatic nuclei. As a result, almost no OsAM1 immunostaining signal was detected in meiocytes of the Osam 1-1 mutant (Figure $8 \mathrm{~A}$ ), indicating that OsAM1 is severely impaired for its localization onto meiotic chromosomes in Osam1-1. We could detect very faint OsAM1 signals occasionally in a few meiocytes. OsREC8 had very weak immunostaining signals in Osam1-1 PMCs (Figure 8B). However, com- 
pared with wild type (Supplementary information, Figure S6), no obvious signals could be detected in Osam 11 PMCs with antibodies against PAIR2, OsMER3 and ZEP1 (Figure 8C-8E), indicating that the loading of these meiotic components might rely on normal functioning of OsAM1. We also performed the same immunostaining in Osam 1-2, and the results were similar to those in Osam 11.

Additionally, we conducted dual immunostaining using both OsAM1 and OsREC8 antibodies in pair2, osmer 3 and zep 1 mutants. In all three mutants, the im- munostaining pattern of both OsREC 8 and OsAM1 appeared to be unaffected (Figure 9). Therefore, we conclude that the installation of OsAM1 and OsREC8 onto chromosomes is independent of PAIR2, OsMER3 and ZEP1.

\section{Discussion}

OsAM1 functions in early meiotic stages

In rice, although lesions in meiosis-specific components can result in various defects in key meiotic pro-

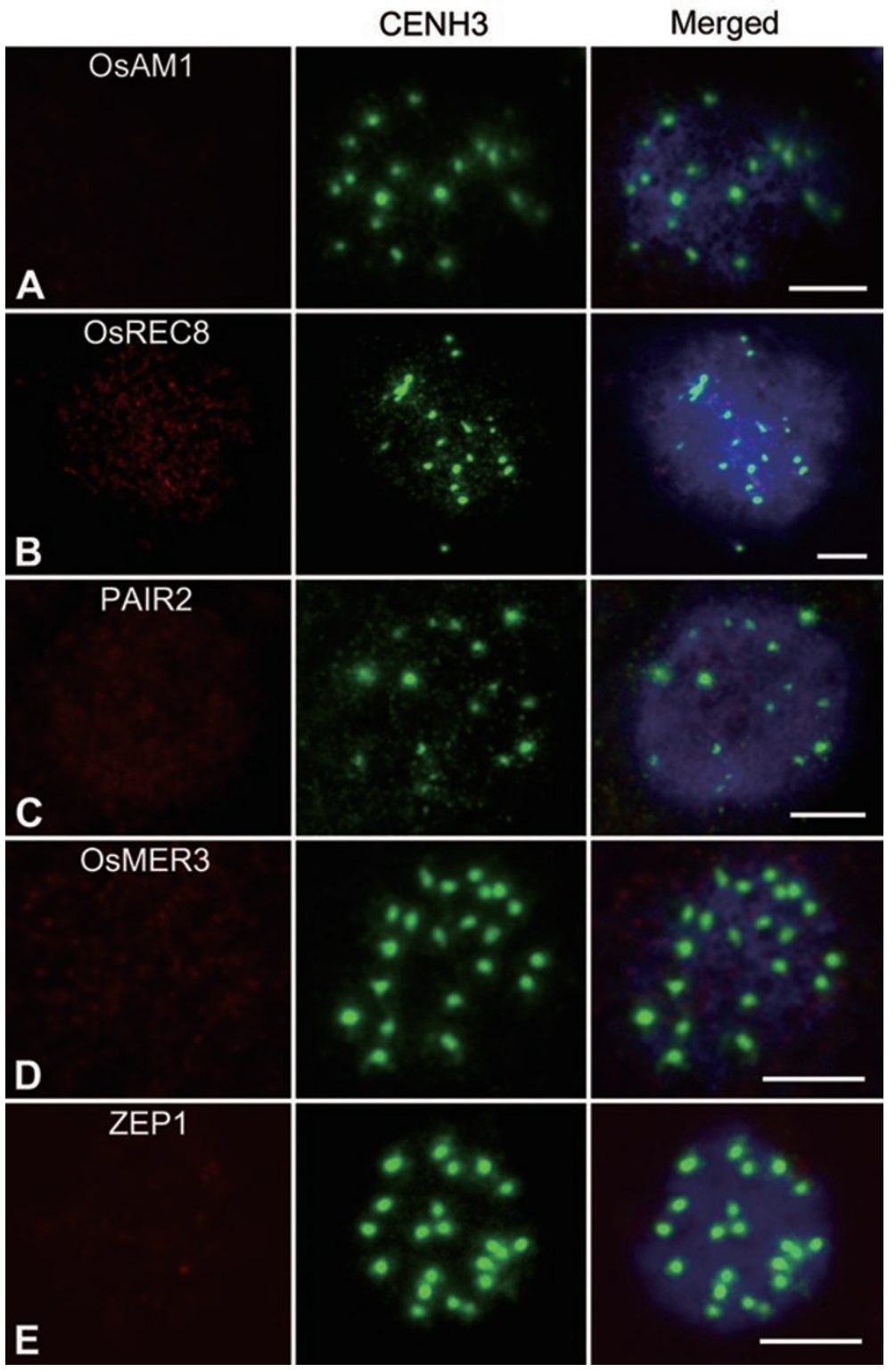

Figure 8 Immunolocalization of several meiotic elements in the Osam1-1 mutant. (A) OsAM1; (B) OsREC8; (C) PAIR2; (D) OsMER3; and (E) ZEP1. An anti-CENH3 antibody is used to indicate the PMCs (the middle panel), and chromosomes are stained with DAPI (blue). Scale bars, $5 \mu \mathrm{m}$. 


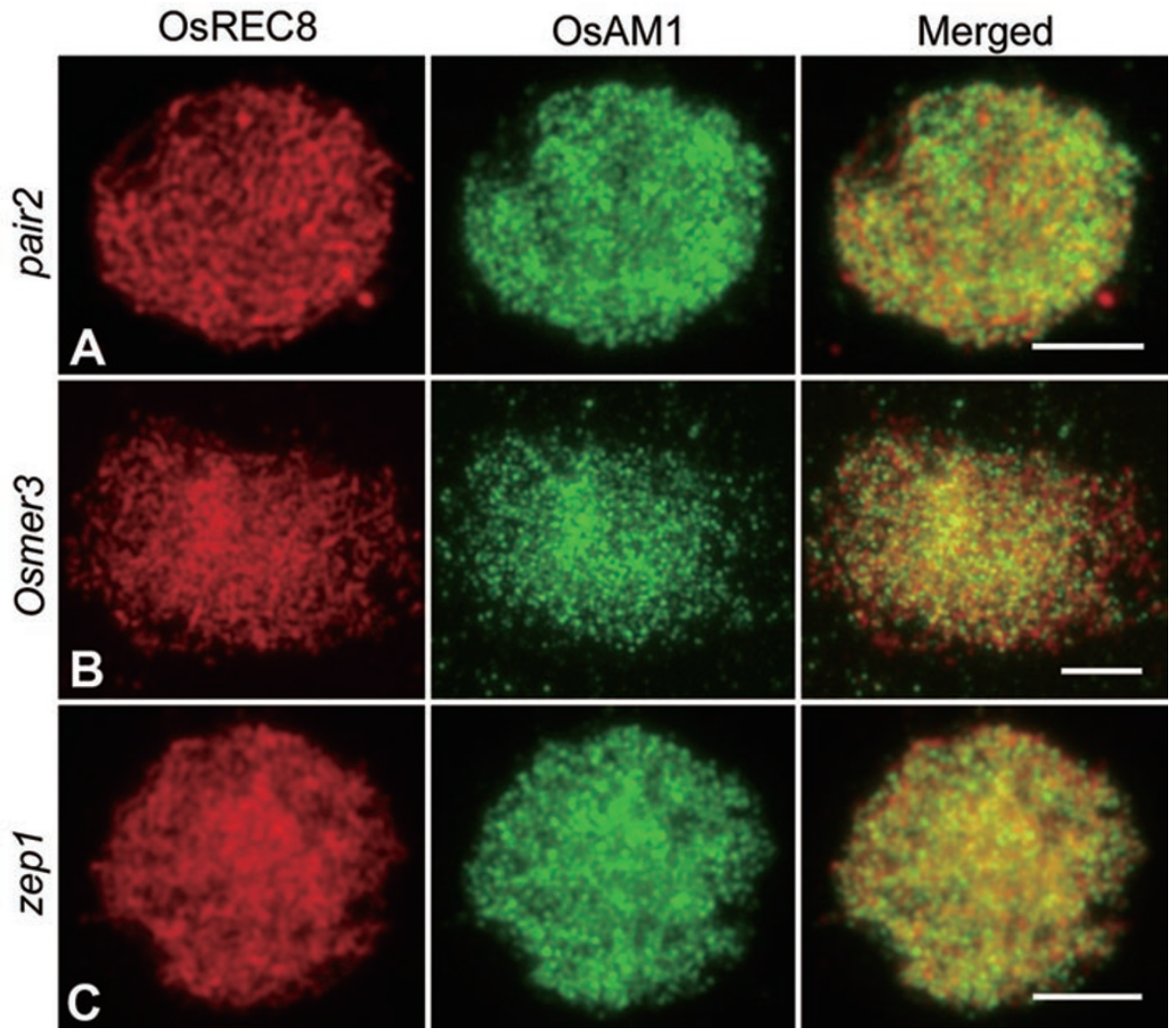

Figure 9 Immunolocalization of OsREC8 and OsAM1 in different rice mutants. (A) Zygotene in pair2; (B) Zygotene in Osmer3; and (C) Zygotene in zep1. Scale bars, $5 \mu \mathrm{m}$.

cesses, they usually do not cause the arrest of meiosis. mell was the first mutant reported that halts the meiotic process in rice [31]. However, in mell, the PAIR2 protein, in addition to its accumulation in the nucleoli, can still associate with chromosome axes. This result implies that AEs in mell may remain intact. Just as in mell, the meiotic process in Osam 1 appears to have initiated, but progression halts in early prophase I. Moreover, the pattern of installation of PAIR2 indicates that assembly of AEs is completely absent in Osam 1 PMCs, demonstrating that the defects of Osam1 are more severe than that of mell. Taken together, these observations indicate that OsAM1 may function at an earlier stage than MEL1 and any other meiotic elements previously reported in rice.

It is known that early meiotic events are interdependent and partially overlapping. For example, in maize, impairments in AFD1 result in defective telomere bouquets, indicating that the elongation of AEs on chromosomes is required for bouquet formation [10]. In rice, installation of PAIR2 depends on the normal formation of AE. In the pair 2 mutant, recombination and synapsis are both lost, suggesting that PAIR2 is indispensable for these important meiotic events. Here, recombina- tion progression and synapsis in $O s a m 1$, as indicated by OsMER3 and ZEP1 signals, are also lost. Therefore, it is probable that the defects in Osam 1 result from the absence of other meiotic elements, an indirect effect of the absence of the OsAM1 protein.

Both OsAM1 and OsREC8 are involved in early meiotic events, but their relationship has not been fully established. OsREC8 is still detected as very faint signals in Osam 1 PMCs, suggesting that the initial recruitment of OsREC8 is independent of OsAM1. This hypothesis is consistent with the fact that OsAM1 and OsREC8 do not show obvious co-localization in wild type at the beginning of prophase I. However, the elongation of OsREC8 signals was impaired in Osam1, suggesting that OsAM1 may facilitate the extension of OsREC8 to form chromosome axes.

Leptotene-zygotene transition is crucial for meiosis progression in rice

Prophase I can be divided into five substages: leptotene, zygotene, pachytene, diplotene and diakinesis. Less is known about the transitions between stages than that of the actual events. This is especially true for the leptotene- 
zygotene transition (also called prezygotene), which is crucial for homologous chromosome pairing and recombination [40]. Maize chromosomes undergo a structural reorganization during the leptotene-zygotene transition [41]: they increase in width and are oval shaped, the sister chromatids separate slightly and the heterochromatin knobs distend. These changes increase the chromosome surface complexity and the area available for interaction with other chromosomes. In addition, some chromosome movements occur at prezygotene: the nucleoli move to the nuclear periphery, pairing of homologous knobs marking telomeres is first observed, telomeres begin to associate with the nuclear envelope and cluster into the bouquet. The movements during this stage may bring distant chromosomes together, facilitating homology search and pairing. In the study of the am1-praI mutant, the authors suggest a novel leptotene-zygotene transition checkpoint, which challenges the former viewpoint that plants lack typical meiotic checkpoints.

Studies in Arabidopsis also show the importance of leptotene-zygotene transition in meiosis [40, 42]. In the ask1-1 mutant, a wide range of defects in male meiosis are detected, such as failure of nucleoli to migrate to the nuclear periphery during prophase I and aberrant synapsis and non-disjunction of homologous chromosomes at anaphase I. Those defects are considered to be a consequence resulting from a block in the leptotene-zygotene transition in ask1-1. ASK1 may have a role in the reorganization of the nucleus at the leptotene-zygotene transition through degradation of some inhibitory proteins in Arabidopsis.

In our study, most chromosomes in Osam 1 PMCs showed the phenotypic characteristics of leptotene, but seemed to stop condensing. The nucleoli remained in the center of nucleus, the bouquet configuration was completely absent and OsREC8 displayed very weak signals. These results indicate that PMCs in Osam1 are halted at the leptotene-zygotene transition. A similar phenomenon of meiotic arrest in maize involving an am1-praI mutant suggests the presence of a novel checkpoint in maize [24], and supports the notion that the leptotene-zygotene transition checkpoint may also exist in rice.

\section{Functional variations among OsAM1, and its Arabidop- sis and maize homologs}

Four swi 1 alleles [20, 21, 43, 44] and six am1 alleles [22-24] have been reported. The two proteins, SWI1 and AM1 have similar functions in the early phases of meiosis, such as regulation of chromosome structure and control of homologous recombination. Moreover, SWI1 and AM1 also share functions during the initiation of meiosis. Mutations in the two genes will cause male and/or female meiocytes to divide in a mitosis-like pattern. However, no swil allele affects the completion of meiosis, indicating that SWI1 may not be involved in meiotic checkpoint control, which discriminates it from AM1. OsAM1 has more similarities to its monocotyledonous homolog AM1. Chromosome behavior of the rice Osam1 mutants seems to be identical to that of maize am1-praI, suggesting that OsAM1 may also be involved in a leptotene-zygotene transition checkpoint. We noted that the am1-praI mutant also carried an arginine-totryptophan substitution, which was very close (four amino acids away) to the mutation point in Osam1-1. Both positions localize to the same crucial domain in AM1like proteins. The OsAM1 kinetics at early prophase I are similar to that of AM1, but we did not observe OsAM1 signals remaining around the centromeres when most of the foci had disappeared from the chromosomes during pachytene [24]. AM1-like proteins may exhibit functional variance even among monocots. Recently, it was reported that a mutation in Arabidopsis SWI1 leads to functional apomeiosis, a major component of apomixes [45]. A similar kind of mutation may also be possible in the rice $O S A M 1$ gene. Rice is one of the most important cereal crops in the world, and apomixis is important for fixation of heterozygosity. Therefore, we hope that Osam 1 alleles with the apomeiosis phenotype may be identified in rice to investigate their potential role in crop improvement.

\section{Materials and Methods}

\section{Plant materials}

The Osam1-1 mutant was found from the japonica rice Wuxiangjing 9 treated with EMS, and the other allele Osam 1-2 was found from the indica rice Zhongxian 3037 induced by ${ }^{60} \mathrm{Co} \sim \gamma$ ray radiation. Three other meiotic mutants, including pair 2 , osmer 3 and zepl, were in this study [29, 33]. All plants were grown in the paddy fields under normal growth condition.

\section{Histological analysis}

Panicles of various developmental stages were fixed in FAA (5\% formaldehyde, $5 \%$ glacial acetic acid, $63 \%$ ethanol) overnight at room temperature. For observation of anther development, the samples were dehydrated through an ethanol series, embedded into Technovit 7100 resin (Heraeus Kulzer) and polymerized at $37^{\circ} \mathrm{C}$. The samples were sectioned into $4-\mu \mathrm{m}$ slices with a Leica microtome and stained with $0.25 \%$ Toluidine blue O (Chroma Gesellshaft Shaud). The slides were photographed with an Olympus BX51 microscope and digital camera.

\section{Molecular cloning of OSAMI}

Based on sequence differences between japonica variety Nipponbare and indica variety 93-11 according to the data published on NCBI website, STS markers (s1-s6) were developed for fine mapping of OsAM. Primers were designed using the PrimerSe- 
lect program of Lasergene (DNASTAR, Madison, WI, USA). All primers mentioned above are listed in Supplementary information, Table S1.

\section{RT-PCR analysis}

Total RNA was isolated from roots, stem, leaf blades, seedlings and young panicles using TRIZOL reagent (Invitrogen) according to the manufacturer's instructions. A measure of $4 \mu \mathrm{g}$ RNA was reverse-transcribed with Oligo-dT(18) primer using M-MLV reverse transcriptase (Promega). The primers OsAM1RTF (5'-TGGTGTAAAAAGGCACATCG-3') and OsAM1RTR (5'-CAGCAGCAATTGTTCCTTCA-3') were designed to examine the expression of OSAM1 in the wild-type and RNAi lines. The same primer pair was used to verify the sequences of AK101644 and AK064367. The standard control Ubiquitin gene was examined with the primers UBI-RTF (5'CAAGATGATCTGCCGCAAATGC-3') and UBI-RTR ( $5^{\prime}$ TTTAACCAGTCCATGAACCCG-3'). RT-PCR analysis was performed with the following profile: $94^{\circ} \mathrm{C}$ for $3 \mathrm{~min}$; 25-30 cycles at $94{ }^{\circ} \mathrm{C}$ for $30 \mathrm{~s}, 57^{\circ} \mathrm{C}$ for $30 \mathrm{~s}$ and $72^{\circ} \mathrm{C}$ for $30 \mathrm{~s}$; and $72{ }^{\circ} \mathrm{C}$ for 10 $\min$.

\section{Generating OsAM1 RNAi transgenic plants}

A 361-bp fragment of OsAM1 was amplified by PCR with the primer pair OsAM1RNAiF (5'-ACTCGAGGCATTACACAACTTCTCCG-3') and OsAM1RNAiR (5'-GAGATCTACTTTGAGTTTGTAACCGAG-3'). The product was ligated on the pMD19-T simple vector (TaKaRa) and then introduced into the BamHI-SalI and BglII-XhoI sites of the pUCCRNAi vector in an inverted repeat orientation. The stem-loop fragment was finally cloned into the pCAMBIA 1300 vector. The RNAi construct was introduced into Agrobacterium tumefaciens strain EHA105 and transformed the japonica cultivar Yandao 8.

\section{Antibody production}

A 213-bp fragment of OsAM1 was amplified from japonica rice panicle cDNA with primers OsAM1GSTF (5'-AAGGATCCAGGCCACAGGTGAGCAGAT-3') and OsAM1GSTR (5' ACCTCGAGATGAATTGGGGATTTGGG-3'), and then ligated to the expression vector pGEX-4T-2 (Amersham) digested with BamHI-XhoI. Then the expression vector was transformed into EScherichia coli strain BL21 (DE3) and was induced by addition of $0.3 \mathrm{mM}$ IPTG to the culture medium at $18{ }^{\circ} \mathrm{C}$.

The OsAM1 fusion peptides were expressed in a soluble fraction and purified using the glutathione Sepharose 4B (GE). Polyclonal antibodies of OsAM1 were raised against a mouse. The PAIR2, OsREC8, OsMER3 and ZEP1 antibodies used in this study were generated previously $[29,33]$. The anti- $\gamma \mathrm{H} 2 \mathrm{AX}$ antibodies were obtained from Upstate Biotechnology (Catalog no. 07-164).

\footnotetext{
Meiotic chromosome preparation, FISH and immunofluorescence

Young panicles of both wild type and mutants were harvested and fixed in Carnoy's solution (ethanol: glacial acetic acid, 3:1) and stored at $-20{ }^{\circ} \mathrm{C}$. Microsporocytes at meiotic stage were squashed and stained with acetocarmine. Slides with chromosome preparations were frozen in liquid nitrogen. After removing the coverslips, the slides were dehydrated through an ethanol series $(70 \%, 90 \%$ and $100 \%)$. Chromosomes were counterstained with
}

4,6-diamidinophenylindole (DAPI) in an antifade solution (Vector Laboratories, Burlingame, CA, USA). Chromosome images were captured under the Olympus BX61 fluorescence microscope with a microCCD camera.

FISH analysis was conducted as described [36]. Two repetitive DNA element probes were used as the FISH probes: pTa794 had the coding sequences for the 5S rRNA genes of wheat [46] and pAtT4 contained the telomeric repeats. Fresh young panicles were fixed in $4 \%(\mathrm{wt} / \mathrm{vol})$ paraformaldehyde for $30 \mathrm{~min}$ at room temperature. Chromosome preparation and immunofluorescence were carried out as previously described [29, 33].

\section{Acknowledgments}

We thank Jason G Walling and Jiming Jiang for critical reading of the manuscript. This work was supported by grants from the Ministry of Sciences and Technology of China (2011CB944602 and 2009ZX08009-068B), and the National Natural Science Foundation of China (30921061 and 31070278).

\section{References}

1 Zickler D, Kleckner N. The leptotene-zygotene transition of meiosis. Annu Rev Genet 1998; 32:619-697.

2 Caryl A, Jones G, Franklin F. Dissecting plant meiosis using Arabidopsis thaliana mutants. J Exp Bot 2003; 54:25-38.

3 Marston A, Amon A. Meiosis: cell-cycle controls shuffle and deal. Nat Rev Mol Cell Biol 2004; 5:983-997.

4 Hamant $\mathrm{O}, \mathrm{Ma} \mathrm{H}$, Cande W. Genetics of meiotic prophase I in plants. Annu Rev Plant Biol 2006; 57:267-302.

5 Klein F, Mahr P, Galova M, et al. A central role for cohesins in sister chromatid cohesion, formation of axial elements, and recombination during yeast meiosis. Cell 1999; 98:91-103.

6 Tanaka K, Watanabe Y. Sister chromatid cohesion and centromere organization in meiosis. Genome Dyn Stab 2008; 2:57-79.

7 Bai X, Peirson B, Dong F, Xue C, Makaroff C. Isolation and characterization of $S Y N 1$, a RAD21-like gene essential for meiosis in Arabidopsis. Plant Cell 1999; 11:417-430.

8 Bhatt A, Lister C, Page T, et al. The DIF1 gene of Arabidopsis is required for meiotic chromosome segregation and belongs to the REC8/RAD21 cohesin gene family. Plant J 1999; 19:463-472.

9 Cai X, Dong F, Edelmann R, Makaroff C. The Arabidopsis $\mathrm{SYN1}$ cohesin protein is required for sister chromatid arm cohesion and homologous chromosome pairing. J Cell Sci 2003; 116:2999-3007.

10 Golubovskaya I, Hamant O, Timofejeva L, et al. Alleles of afd1 dissect REC8 functions during meiotic prophase I. $J$ Cell Sci 2006; 119:3306-3315.

11 Zhang L, Tao J, Wang S, Chong K, Wang T. The rice Os$\operatorname{Rad} 21-4$, an orthologue of yeast $\operatorname{Rec} 8$ protein, is required for efficient meiosis. Plant Mol Biol 2006; 60:533-554.

12 Chelysheva L, Diallo S, Vezon D, et al. AtREC8 and AtSCC3 are essential to the monopolar orientation of the kinetochores during meiosis. J Cell Sci 2005; 118:4621-4632.

13 Franklin A, McElver J, Sunjevaric I, Rothstein R, Bowen B, Cande W. Three-dimensional microscopy of the Rad51 recombination protein during meiotic prophase. Plant Cell 
1999; 11:809-824.

14 Pawlowski W, Golubovskaya I, Cande W. Altered nuclear distribution of recombination protein RAD51 in maize mutants suggests the involvement of RAD51 in meiotic homology recognition. Plant Cell 2003; 15:1807-1816.

15 Pawlowski W, Golubovskaya I, Timofejeva L, Meeley R, Sheridan W, Cande W. Coordination of meiotic recombination, pairing, and synapsis by PHS1. Science 2004; 303:8992.

16 Harper L, Golubovskaya I, Cande W. A bouquet of chromosomes. J Cell Sci 2004; 117:4025-4032.

17 Golubovskaya I, Harper L, Pawlowski W, Schichnes D, Cande W. The pam 1 gene is required for meiotic bouquet formation and efficient homologous synapsis in maize (Zea mays L.). Genetics 2002; 162:1979-1993.

18 Watanabe Y, Yokobayashi S, Yamamoto M, Nurse P. Pre-meiotic $\mathrm{S}$ phase is linked to reductional chromosome segregation and recombination. Nature 2001; 409:359-363.

19 Pawlowski W, Sheehan M, Ronceret A. In the beginning: the initiation of meiosis. Bioessays 2007; 29:511-514.

20 Motamayor J, Vezon D, Bajon C, et al. Switch (swil), an Arabidopsis thaliana mutant affected in the female meiotic switch. Sex Plant Reprod 2000; 12:209-218.

21 Mercier R, Vezon D, Bullier E, et al. SWITCH1 (SWI1): a novel protein required for the establishment of sister chromatid cohesion and for bivalent formation at meiosis. Genes Dev 2001; 15:1859-1871.

22 Golubovskaya I, Grebennikova Z, Avalkina N, Sheridan W. The role of the ameioticl gene in the initiation of meiosis and in subsequent meiotic events in maize. Genetics 1993; 135:1151-1166.

23 Golubovskaya I, Avalkina N, Sheridan W. New insights into the role of the maize ameioticl locus. Genetics 1997; 147:1339-1350.

24 Pawlowski W, Wang C, Golubovskaya I, et al. Maize AMEIOTIC1 is essential for multiple early meiotic processes and likely required for the initiation of meiosis. Proc Natl Acad Sci USA 2009; 106:3603-3608.

25 Mercier R, Armstrong S, Horlow C, et al. The meiotic protein SWI1 is required for axial element formation and recombination initiation in Arabidopsis. Development 2003; 130:33093318.

26 Jenkins G, Phillips D, Mikhailova E, Timofejeva L, Jones R. Meiotic genes and proteins in cereals. Cytogenet Genome Res 2008; 120:291-301.

27 Nonomura K, Nakano M, Fukuda T, et al. The novel gene HOMOLOGOUS PAIRING ABERRATION IN RICE MEIO$S I S 1$ of rice encodes a putative coiled-coil protein required for homologous chromosome pairing in meiosis. Plant Cell 2004; 16:1008-1020.

28 Nonomura K, Nakano M, Murata K, et al. An insertional mutation in the rice PAIR2 gene, the ortholog of Arabidopsis $A S Y 1$, results in a defect in homologous chromosome pairing during meiosis. Mol Genet Genomics 2004; 271:121-129.
29 Wang K, Tang D, Wang M, et al. MER3 is required for normal meiotic crossover formation, but not for presynaptic alignment in rice. $J$ Cell Sci 2009; 122:2055-2063.

30 Yuan W, Li X, Chang Y, et al. Mutation of the rice gene PAIR 3 results in lack of bivalent formation in meiosis. Plant $J$ 2009; 59:303-315.

31 Nonomura K, Morohoshi A, Nakano M, et al. A germ cell specific gene of the ARGONAUTE family is essential for the progression of premeiotic mitosis and meiosis during sporogenesis in rice. Plant Cell 2007; 19:2583-2594.

32 Deng Z, Wang T. OsDMC1 is required for homologous pairing in Oryza sativa. Plant Mol Biol 2007; 65:31-42.

33 Wang M, Wang K, Tang D, et al. The central element protein ZEP1 of the synaptonemal complex regulates the number of crossovers during meiosis in rice. Plant Cell 2010; 22:417430.

34 Feng J, Lu Y, Liu X, Xu X. Pollen development and its stages in rice (Oryza sativa L.). Chinese J Rice Sci 2001; 15:21-28.

35 Itoh J, Nonomura K, Ikeda K, et al. Rice plant development: from zygote to spikelet. Plant Cell Physiol 2005; 46:23-47.

36 Zhang W, Yi C, Bao W, et al. The transcribed 165-bp CentO satellite is the major functional centromeric element in the wild rice species Oryza punctata. Plant Physiol 2005; 139:306-315.

37 Hunter N, Börner G, Lichten M, Kleckner N. $\gamma$-H2AX illuminates meiosis. Nat Genet 2001; 27:236-238.

38 Sanchez-Moran E, Santos J, Jones G, Franklin F. ASY1 mediates AtDMC1-dependent interhomolog recombination during meiosis in Arabidopsis. Genes Dev 2007; 21:2220-2233.

39 Nonomura K, Nakano M, Eiguchi M, Suzuki T, Kurata N. PAIR2 is essential for homologous chromosome synapsis in rice meiosis I. J Cell Sci 2006; 119:217-225.

40 Wang Y, Wu H, Liang G, Yang M. Defects in nucleolar migration and synapsis in male prophase I in the ask1-1 mutant of Arabidopsis. Sex Plant Reprod 2004; 16:273-282.

41 Dawe R, Sedat J, Agard D, Cande W. Meiotic chromosome pairing in maize is associated with a novel chromatin organization. Cell 1994; 76:901-912.

42 Yang X, Timofejeva L, Ma H, Makaroff C. The Arabidopsis SKP1 homolog ASK1 controls meiotic chromosome remodeling and release of chromatin from the nuclear membrane and nucleolus. J Cell Sci 2006; 119:3754-3763.

43 Boateng K, Yang X, Dong F, Owen H, Makaroff C. SWI1 is required for meiotic chromosome remodeling events. Mol Plant 2008; 1:620-633.

44 Siddiqi I, Ganesh G, Grossniklaus U, Subbiah V. The dyad gene is required for progression through female meiosis in Arabidopsis. Development 2000; 127:197-207.

45 Ravi M, Marimuthu M, Siddiqi I. Gamete formation without meiosis in Arabidopsis. Nature 2008; 451:1121-1124.

46 Cuadrado A, Jouve N. Mapping and organization of highlyrepeated DNA sequences by means of simultaneous and sequential FISH and C-banding in 6x-triticale. Chromosome Res 1994; 2:331-338.

(Supplementary information is linked to the online version of the paper on the Cell Research website.) 